\title{
Stratified treatment recommendation or one-size-fits-all? A health economic insight based on graphical exploration
}

\author{
Qi Cao ${ }^{1,2}$ (]) Erik Buskens ${ }^{2} \cdot$ Hans L. Hillege $\mathrm{e}^{2,3} \cdot$ Tiny Jaarsma $^{4} \cdot$ Maarten Postma $^{1,2,5,6} \cdot$ Douwe Postmus $^{2}$
}

Received: 17 January 2018 / Accepted: 23 October 2018 / Published online: 29 October 2018

(c) The Author(s) 2018

\begin{abstract}
Objectives We sought to explore to what extent the use of Subpopulation Treatment Effect Pattern Plot (STEPP) may help to identify efficient treatment allocation strategy.

Methods The analysis was based on data from the COACH study, in which 1023 patients with heart failure were randomly assigned to three treatments: care-as-usual, basic support, and intensive support. First, using predicted 18-month mortality risk as the stratification basis, a suitable strategy for assigning different treatments to different risk groups of patients was developed. To that end, a graphical exploration of the difference in net monetary benefit (NMB) across treatment regimens and baseline risk was used. Next, the efficiency gains resulting from this proposed subgroup strategy were quantified by computing the difference in NMB between our stratified approach and the best performing population-wide strategy.

Results The analysis using STEPPs suggested that a differentiated approach, based on offering intensive support to lowrisk patients $(18$-month mortality risk $\leq 0.16)$ and basic support to intermediate- to high-risk patients $(18$-month mortality risk $>0.16$ ) would be an economically efficient treatment allocation strategy. This was confirmed in the subsequent costeffectiveness analysis, where the average gain in NMB resulting from the proposed stratified approach compared to basic support for all was found to be $€ 1312$ (95\% CI €390-€2346) per patient.

Conclusions STEPP provides a systematic approach to assess the interaction between baseline risk and the difference in NMB between competing interventions and to identify cutoffs to stratify patients in a health economically optimal manner.
\end{abstract}

Keywords Cost-effectiveness $\cdot$ Risk stratification $\cdot$ Personalized medicine $\cdot$ Heart failure

JEL $\mathrm{C} 02 \cdot \mathrm{C} 10 \cdot \mathrm{I} 10 \cdot \mathrm{I} 12$

Qi Cao

q.cao@rug.nl

1 Unit of PharmacoTherapy, -Epidemiology and -Economics, Groningen Research Institute of Pharmacy (GRIP), University of Groningen, Antonius Deusinglaan 1, 9713 AV Groningen, The Netherlands

2 Department of Epidemiology, University of Groningen, University Medical Center Groningen, Hanzeplein 1, 9713 GZ Groningen, The Netherlands

3 Department of Cardiology, University of Groningen, University Medical Center Groningen, Groningen, Hanzeplein 1, 9713 GZ Groningen, The Netherlands
4 Department of Social and Welfare Studies, Faculty of Health Sciences, Linköping University, 581 83, Linköping, Sweden

5 Institute for Science in Healthy Aging and healthcaRE (SHARE), University of Groningen, University Medical Center Groningen (UMCG), Hanzeplein 1, 9713 GZ Groningen, The Netherlands

6 Department of Health Sciences, University of Groningen, University Medical Center Groningen (UMCG), Hanzeplein 1, 9713 GZ Groningen, The Netherlands 


\section{Introduction}

Cost-effectiveness analysis supports resource allocation decision making by comparing the differences in costs and effects of alternative treatment regimens [1-4]. When such analyses are conducted alongside randomized controlled trials (RCTs), the cost-effectiveness of the evaluated treatments is generally expressed in terms of population averages. This provides insight into which of the available treatments performs best for the patient population considered. However, when these patients are characterized by a heterogeneous clinical condition, and their risk profiles are determined by factors like demographic variations, biometric variations, and co-morbidities, there may be considerable variation in response. In fact, the likelihood of subpopulations for whom response to one or the other treatment is obscured may be substantial [5-8]. Such differences among patients may also lead to systematic variation in resource use and costs, which could be another reason why one of the other treatments performs better in specific subpopulations [2, 6]. Acknowledging patient heterogeneity in health economic evaluation has therefore considerable potential in more efficient resource allocation decision-making [5, 8-10].

A recently conducted systematic review [5] identified baseline risk, treatment effect, health state utility, and resource utilization as the four input parameters of a health economic evaluation that may be prone to patient heterogeneity. However, as the cost-effectiveness of one treatment compared to another is ultimately determined by the net effect on all these parameters, it is essential that the impact of patient heterogeneity on each of these parameters is considered conjointly rather than in isolation, especially when the purpose is to identify more efficient reimbursement policies. For health economic evaluations conducted alongside an RCT, this can be achieved by conducting such analyses directly in terms of net monetary benefit (NMB) [11-13].

Hoch et al. [14] have previously proposed assessing the impact that different sources of patient heterogeneity may have on a treatment's NMB by means of regression analysis. For example, suppose that one wants to explore whether the cost-effectiveness of a new treatment compared to the current standard treatment is affected by the age of the patient. Using regression analysis, this can be achieved by fitting a regression model with NMB as the dependent variable and the treatment indicator, age, and the interaction between age and the treatment indicator as the independent variables. A low $p$ value for the regression coefficient corresponding to the interaction term then shows that age has a relatively strong influence on the new treatment's relative cost-effectiveness.

While the use of multivariable regression models may provide insight into which sources of patient heterogeneity potentially have an impact on the relative cost-effectiveness of the evaluated treatments, the statistical power to detect such interaction effects is usually low. Moreover, actually being able to verify relevant heterogeneity using such models strongly depends on whether the assumed multiplicative structure of interaction fits reality. This may lead to missing or over-interpretation of the detected significant interaction terms. An alternative approach for studying treatment-covariate interaction that makes no assumptions about the nature of the relationship between the outcome and the covariate in each treatment group is the Subpopulation Treatment Effect Pattern Plot (STEPP) methodology [15-17]. This is based on a graphical exploration of the fluctuation in treatment effect across different, but overlapping subpopulations defined with respect to increasing levels of the covariate of interest. Although using STEPP to explore how the difference in NMB between two treatments varies as a function of one or more sources of patient heterogeneity could potentially be very useful in identifying more efficient reimbursement policies, to the best of our knowledge, it has not yet been considered. Using the difference in NMB as the measure of treatment benefit and an individualized predicted risk obtained from an RCT as the covariate of interest, the objective of this paper was to illustrate how the STEPP methodology can be used to derive risk-stratified treatment allocation strategies that maximize cost-effectiveness. Specifically, a case study in heart failure (HF) disease management was elaborated.

\section{Methods}

\section{Study cohort}

The data that we used to conduct our analysis was taken from the Coordinating study evaluating Outcomes of Advising and Counseling in Heart failure (COACH), a multicenter RCT in which 1023 patients were randomly assigned to one of three disease management programs (DMPs) [18, 19]. Patients in the care-as-usual group received routine followup management by a cardiologist. Along with this routine management, patients in the basic and intensive support groups received additional care from an HF nurse. In addition, patients in the intensive support group received multidisciplinary advice and two or more home visits by the HF nurse. The total follow-up time of the trial was 18 months.

\section{Baseline risk assessment}

The patients' predicted 18-month all-cause mortality risk was obtained from a previously developed multivariable risk prediction model [20]. This model included the following 14 predictor variables: age, gender, diastolic blood pressure, 
systolic blood pressure, history of stroke, history of myocardial infarction, atrial fibrillation, peripheral arterial disease, diabetes, left ventricular ejection fraction, previous HF hospitalization, serum sodium, estimated glomerular filtration rate (eGFR), and N-terminal pro brain natriuretic peptide (NT-proBNP). Missing values on these predictor variables were dealt with using multiple imputation [21]. Mortality risk values were then computed by taking the average of the risk values obtained from each of the ten imputed datasets.

\section{Patient-level NMB assessment}

The patient-level NMB was calculated as $\mathrm{NMB}_{i}=\lambda \times e_{i}-c_{i}$, where $e_{i}$ and $c_{i}$ denote the observed effect and cost for patient $i$, and $\lambda$ denotes the willingness-to-pay threshold [14]. The patients' observed survival time, which was censored at 18 months for those who were still alive at the end of the study's follow-up, was taken as the measure of effectiveness. Costs were calculated at the patient level by multiplying the patients' volumes of resource use with their respective unit costs as described in more detail in a previously conducted economic evaluation in this patient population [22]. The willingness-to-pay threshold was set equal to $€ 20,000$ per life year, which is the same threshold as was used in the aforementioned study [22].

\section{Exploration of treatment-predicted risk interaction and determination of subgroup strategy}

To explore whether an interaction existed between the COACH DMPs and the predicted 18-month mortality risk, we applied the STEPP methodology $[15,16]$ using the difference in NMB as the outcome of interest. STEPP is a novel graphical method for assessing treatment-covariate interaction on different, but overlapping subpopulations defined with respect to the covariate of interest. The subpopulations are defined on the basis of two parameters: (1) the number of patients in common among consecutive subpopulations $\left(n_{1}\right)$ and (2) the number of patients in each subpopulation $\left(n_{2}\right)$. As the estimation of the interaction effect varies for different combinations of the parameter values, it is recommended to repeat the analysis for different values of $n_{1}$ and $n_{2}$ until the interaction effect stabilizes (i.e., a similar pattern is shown in the graph) [23]. For the analysis conducted in this study, stable estimates of the interaction effects were obtained by setting $n_{1}$ and $n_{2}$ equal to 120 and 150 , respectively. Specifically, this means that the first subpopulation consisted of the 150 patients with the lowest predicted 18 -month mortality risks. To obtain the next subpopulation of 150 patients, the 30 patients with the lowest mortality risks were replaced by the 30 patients with the next highest mortality risks. This process was repeated until all patients were included in at least one of the subpopulations. For this study, two STEPPs were created: one with the difference in NMB between careas-usual and basic support as the outcome and another with the difference in NMB between intensive support and basic support as the outcome. Basic support was selected as the reference category because it was previously shown to be the optimal population-wide strategy [22]. Based on the observed patterns of treatment-predicted risk interaction, a suitable strategy for assigning different DMPs to different risk groups of patients was subsequently identified.

\section{Quantification of the efficiency gains resulting from the subgroup strategy}

To evaluate the optimality of our proposed subgroup strategy, we quantified its efficiency gains as suggested by Coyle et al. [8]. First, the average NMB was evaluated separately per DMP for each of the established risk categories. Subsequently, the average gain in NMB resulting from stratification compared to the best performing population-wide strategy was calculated as $\overline{\mathrm{NMB}}=\sum_{j} \frac{\Delta \mathrm{NMB}_{j} \times n_{j}}{N}$, where $\Delta \mathrm{NMB}_{j}$ denotes the difference in average NMB between the proposed treatment for subgroup $j$ and the best performing population-wide strategy (basic support in our case), $n_{j}$ denotes the sample size of subgroup $j$, and $N$ denotes the sample size of the overall study population. In the previously conducted economic evaluation, it was suggested that the New York Heart Association (NYHA) class, which is a generally used functional classification to describe the severity of HF symptoms, could be a suitable basis for offering different treatments to different subgroups of patient. For comparative purposes, the average gain in NMB resulting from using NYHA class as the stratification basis was computed as well. 95\% confidence intervals (CIs) for the efficiency gain estimates were obtained through bootstrapping.
Table 1 Patient-level mortality risk and NMB estimates stratified by the three DMP groups

\begin{tabular}{lll}
\hline Patient subgroup (sample size) & $\begin{array}{l}\text { Median (IQR) 18-month all-cause } \\
\text { mortality risk }\end{array}$ & Median (IQR) NMB $(€)$ \\
\hline Care-as-usual $(n=339)$ & $0.25(0.14-0.40)$ & $22,880(3544-29,012)$ \\
Basic support $(n=340)$ & $0.22(0.14-0.35)$ & $24,146(7253-28,573)$ \\
Intensive support $(n=344)$ & $0.24(0.12-0.37)$ & $24,064(5256-28,208)$ \\
\hline
\end{tabular}

$I Q R$ inter-quartile range 


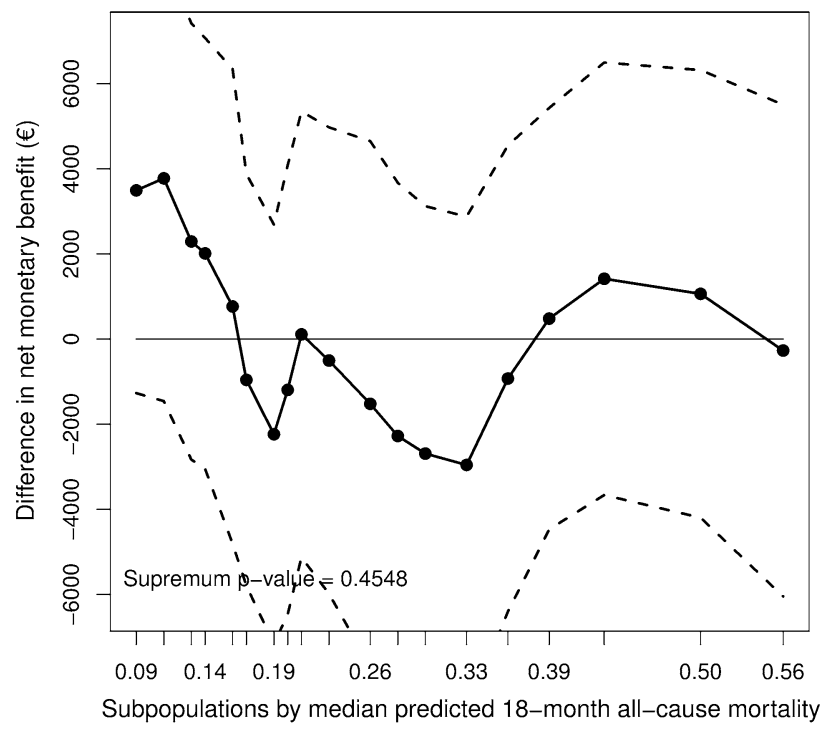

Fig. 1 STEPP comparing the difference in NMB between care-asusual and basic support across different, but overlapping subpopulations with increased mortality risk; a difference in NMB $>0$ indicates that care-as-usual is the preferred strategy

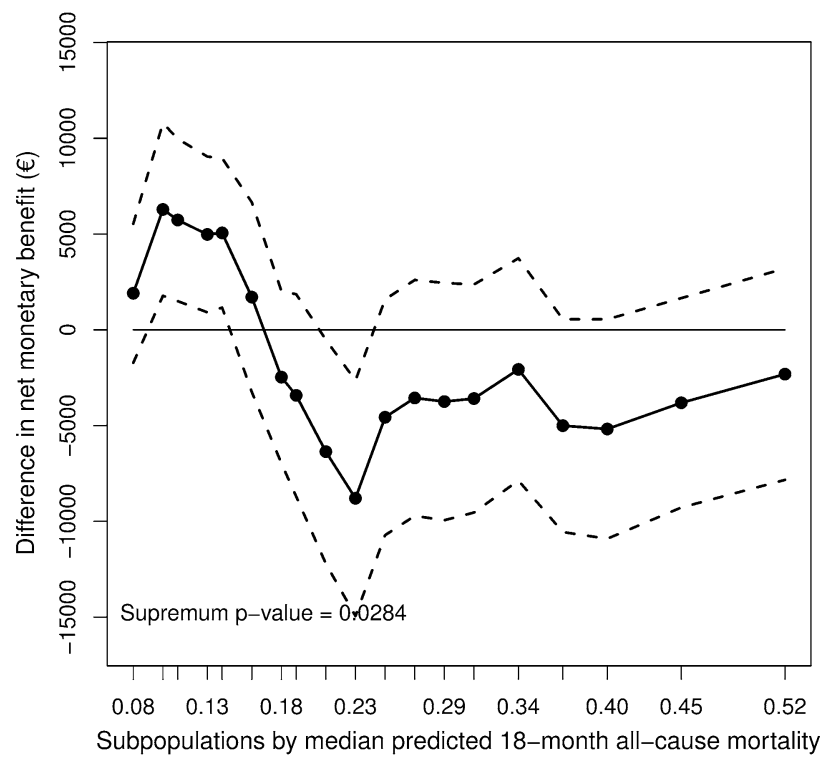

Fig. 2 STEPP comparing the difference in NMB between intensive support and basic support across different, but overlapping subpopulations with increased mortality risk; a difference in NMB $>0$ indicates that intensive support is the preferred strategy

\section{Results}

\section{Patient-level mortality risk and NMB estimates}

The median of the patient-level 18-month all-cause mortality risk estimates was 0.23 (inter-quartile range: $0.14-0.37$ ) with a minimum value of 0.01 and a maximum value of 0.93 . Table 1 summarizes the distribution of the patient-level mortality risk and NMB values stratified by the three different DMP groups. It shows that patients from the basic support group had the lowest median mortality risk and the highest median NMB when the willingness-to-pay threshold was set to $€ 20,000$. This is consistent with the previously conducted economic evaluation [22] where basic support was found to be the optimal one-size-fits-all strategy.

\section{Exploration of treatment-predicted risk interaction and determination of subgroup strategy}

The estimated difference in NMB across the overlapping patient subpopulations are depicted in Figs. 1 and 2. The pattern of the difference in NMB between care-as-usual and basic support does not suggest clear cutoff points to stratify patients into different risk groups (Fig. 1). In addition, the fact that the treatment-covariate interaction effect was not significant ( $p$ value $>0.05$ ), suggests that patient heterogeneity did not have a clear impact on the difference in NMB between these two treatments. However, a significant treatment-covariate interaction was found when comparing the difference in NMB between intensive support and basic support (Fig. 2). In addition, the pattern depicted in the plot suggests a risk value of 0.16 to be the zero point at which the difference in NMB between these two treatments starts to change signs. Based on this finding, our proposed subgroup strategy was to assign intensive support to low-risk patients (patients with predicted risk value $\leq 0.16$ ) and basic support to intermediate- to high-risk patients (patients with predicted risk value $>0.16$ ).

\section{Subgroup cost-effectiveness results}

Table 2 depicts the results of the cost-effectiveness analysis within each risk stratum. For the low-risk patients, intensive support was found to be the best performing strategy with the highest amount of NMB, while basic support performed best in the intermediate- to high-risk patients. When NYHA class was used as the stratification basis, basic support was found to be optimal for less severe patients (i.e., those belonging to NYHA class II), while care-as-usual was found to be optimal for severe patients (i.e., those belonging to NYHA class III and IV).

\section{Quantification of the efficiency gains resulting from the subgroup strategy}

Table 3 depicts the average gains in NMB $(95 \% \mathrm{CI})$ resulting from each subgroup strategy. Both strategies were found to be cost-effective compared to assigning basic support to 
Table 2 Results of the cost-effectiveness analysis

\begin{tabular}{|c|c|c|c|}
\hline Patient subgroup (sample size) & $\begin{array}{l}\text { Mean }(95 \% \text { CIs) survival time } \\
\text { (days) }\end{array}$ & Mean (95\% CIs) cost $(€)$ & Mean $(95 \%$ CIs) NMB $(€)$ \\
\hline \multicolumn{4}{|l|}{ Predicted 18-month mortality } \\
\hline \multicolumn{4}{|l|}{ Risk $\leq 0.16(N=321)$} \\
\hline Care-as-usual & $521.2(497.5-543.6)$ & $6151(3961-8826)$ & $22,389(19,200-25,101)$ \\
\hline Basic support & $525.6(505.2-545.5)$ & $8653(6117-11,664)$ & $20,127(17,107-22,844)$ \\
\hline Intensive support & $557.2(547.7-562.0)$ & $6213(4950-7624)$ & $24,307(22,857-25,577)$ \\
\hline \multicolumn{4}{|l|}{ Risk $>0.16(N=702)$} \\
\hline Care-as-usual & $428.2(403.2-451.6)$ & $11,175(9348-13,226)$ & $12,265(9791-14,496)$ \\
\hline Basic support & $454.0(429.6-480.5)$ & $10,041(8257-11,935)$ & $14,819(12,608-17,071)$ \\
\hline Intensive support & $432.3(406.1-456.9)$ & $13,155(11,221-15,142)$ & $10,525(7935-12,803)$ \\
\hline \multicolumn{4}{|l|}{ NYHA class } \\
\hline \multicolumn{4}{|l|}{ NYHA II $(N=513)$} \\
\hline Care-as-usual & $481.4(455.9-504.5)$ & $8955(6884-11,522)$ & $17,405(14,318-20,026)$ \\
\hline Basic support & $506.6(486.0-528.6)$ & 7170 (5788-8898) & $20,570(18,607-22,250)$ \\
\hline Intensive support & $505.7(484.8-527.0)$ & $9099(7256-11,220)$ & $18,581(16,087-20,758)$ \\
\hline \multicolumn{4}{|l|}{ NYHA III and IV $(N=495)$} \\
\hline Care-as-usual & $428.7(397.0-462.3)$ & $10,692(8279-13,206)$ & $12,788(10,112-15,948)$ \\
\hline Basic support & $443.7(414.7-471.1)$ & $11,793(9435-14,403)$ & $12,507(9465-15,219)$ \\
\hline Intensive support & $448.9(422.2-474.8)$ & $12,462(10,279-14,779)$ & $12,118(9304-14,707)$ \\
\hline
\end{tabular}

Table 3 Average gains in NMB (95\% CIs) resulting from each subgroup strategy

\begin{tabular}{lll}
\hline Stratification basis & Subgroup strategy & $\begin{array}{l}\text { Average (95\% CIs) } \\
\text { gain in NMB (€) }\end{array}$ \\
\hline Predicted 18-month mortality & $\begin{array}{l}\text { Intensive support to low-risk group } \\
\text { Basic support to intermediate- to high-risk } \\
\text { group }\end{array}$ & 1312 (390 to 2346) \\
& $\begin{array}{l}\text { Basic support to NYHA II group } \\
\text { Care-as-usual to NYHA III and IV group }\end{array}$ & 138 (-1854 to 2246) \\
& &
\end{tabular}

the whole patient population. However, the subgroup strategy proposed in this study outperformed the one proposed previously, with an average gain in NMB of $€ 1174$ (95\% CI $€-1146-€ 3284)$.

\section{Discussion}

STEPP is a relatively new approach to graphically explore treatment-covariate interaction with limited application in the clinical field [23-26]. By using STEPP to graphically explore treatment-covariate interaction, we found that the difference in NMB between intensive support and basic support varied greatly across different, but overlapping subpopulations defined with respect to increasing levels of predicted 18-month mortality risk. The difference in NMB between care-as-usual and basic support, in contrast, never led to a clear pattern of treatment-covariate interaction. By subsequently selecting the 18-month mortality risk at which the difference in NMB between intensive support and basic support started to change signs as the cutoff to stratify patients into two risk categories, we found that compared to applying basic support to all patients, the use of a stratified approach based on offering intensive support to lowrisk patients and basic support to intermediate- to high-risk patients would result in an average gain in NMB of $€ 1312$ (95\% CI €390-€2346).

Our finding that more intensive multidisciplinary disease management is not beneficial in intermediate- to high-risk patients may seem counterintuitive to some readers, but is consistent with the study conducted by Pulignano et al. [27], who concluded that "most eligible patients for a hospitalbased DMP may be those at intermediate risk who are not too sick and not too healthy". Our STEPP for care-as-usual against basic support suggests that this also holds for the moderate form of disease management that was provided in the COACH study. However, our other STEPP indicates that, compared to basic support, low-risk patients may still benefit from a more intensive form of disease management. Although there is also evidence to suggest that intensive, 
post-discharge disease management is unnecessary in lowrisk patients [28-30], our latter finding is consistent with several previously conducted subgroup analyses. Hebert et al. [31] found that when comparing severe (NYHA class III and IV) and less severe (NYHA class I and II) patients, nurse-led disease management was more likely to be costeffective in the less severe patients. Similarly, Miller et al. [32], who conducted a model-based evaluation to investigate the lifetime cost-effectiveness of telephonic support for systolic HF patients, obtained a slightly less favorable cost-effectiveness ratio for this intervention after NYHA class I patients were eliminated from their study population. Finally, Goehler et al. [33] found that the median lifetime incremental cost-effectiveness ratio increased with $€ 15,900$ / quality-adjusted life year (QALY) for male patients and $€ 600 / \mathrm{QALY}$ for female patients when the average age of the cohort passing through their model was increased from 55 to 75 years. When combining our results with the findings presented in these previous studies, it seems that the trade-off between a moderate or intensive form of disease management is shown especially in patients at low or intermediate risk who are not too sick to be treated. Patients at high risk, in contrast, do not seem to benefit from a more intense form of multidisciplinary disease management. The question of whether such patients should therefore only be offered a basic form of disease management is an ethical discussion that is beyond the scope of this paper.

In our analysis, we applied a previously developed multivariable risk prediction model to combine the information captured within several covariates into a single prognostic index to represent baseline risk. We subsequently used this index to explore for heterogeneity in treatment effect across different subgroups of patients. Compared to conventional subgroup analysis based on a single prognostic covariate, integrating multiple independent patient characteristics associated with the outcome parameters of interest in a multivariable risk prediction model improves risk stratification $[34,35]$. This, in turn, can greatly enhance the statistical power to detect variations in treatment benefit as was shown in a previously conducted simulation study [36]. Moreover, the use of such a multivariable approach avoids the problem of multiple testing, resulting from the need to repeat the subgroup analysis for different individual risk factors. Thus, the chances of obtaining false positive findings are reduced [36, 37].

While treatment-predicted risk interaction can best be assessed on a continuous scale [38], discretization of the predicted risks into two or more ordinal categories becomes essential if we want to use the underlying risk prediction model to guide the selection of therapy. By deriving the cutoff of 0.16 from the treatment effect pattern observed in a STEPP, we were still able to make effective use of the discriminative power of a continuous prognostic index in our quest for an efficient reimbursement policy. This does not hold when applying conventional subgroup analysis based on a single prognostic covariate as we did as part of our previous economic evaluation in this patient population [22]. When quantifying the net benefit gains of one over the other stratification basis, the subgroup strategy proposed in this study was found to outperform the previous one with an average gain in NMB of $€ 1174$ (95\% CI $€-1146$ to $€ 3284$ ).

A limitation of this study is that the cutoff of 0.16 may be specific for the data analyzed in this paper. It was selected by taking into account the pattern of treatment-risk interaction in a single clinical trial. Future research is thus required to determine to what extent this cutoff can also serve as a suitable stratification basis for other studies. Secondly, rather than using an external model (i.e., a risk prediction model developed on another dataset), we used an internally developed risk prediction model to assess the treatment effect across different subpopulations of predicted risk. The validity of this approach was recently assessed by Burke et al. [34], who concluded that "appropriately developed internal models produce relatively unbiased estimates of treatment effect across the spectrum of risk". In addition, these authors also found that "when estimating treatment effect, internally developed risk models using both treatment arms should, in general, be preferred to models developed on the control population". As all treatment groups of $\mathrm{COACH}$ were included in the development of the $\mathrm{COACH}$ risk prediction model, this is exactly the strategy that we have followed in the current paper. Thirdly, because we selected the difference in NMB as the measure of treatment benefit, our results are conditional on the value assumed for the willingnessto-pay threshold. As a first paper to introduce the application of our proposed approach, we only selected a single threshold. For actual decision-making purposes, it would however be recommended to perform sensitivity analysis and repeat the approach for different values of the willingness-to-pay threshold to make sure that the risk-stratified treatment recommendation is robust with respect to the selected threshold value. Another limitation of this study is that the time horizon for the economic evaluation was restricted to the 18-month follow-up period of the $\mathrm{COACH}$ study, meaning that cost differences and survival benefits are likely to be underestimated. In future applications of our proposed method, one could therefore consider extrapolating the patient-level cost and survival estimates beyond the range of the trial data by applying more advanced statistical modeling techniques, such as the multi-state modeling approach proposed by Cao et al. [39]. Finally, heterogeneity in individual patient preferences was not considered in our analysis, although it was suggested as being an important factor when developing personalized treatment recommendations [5]. 
To conclude, the emerging role of health economics in personalized medicine has recently been recognized and is actively discussed [40-45]. To assess how personalized medicine may maximize the net benefits, it is crucial to develop a risk-stratified treatment recommendation [46] to ensure subgroup cost-effectiveness analysis. Recently, value of information analysis was adapted to develop stratified treatment recommendations that maximize net health benefit or NMB $[9,10]$. This technique may be useful when a model-based economic evaluation is conducted. Our proposed approach based on STEPP enables the development of stratified treatment recommendations when the economic evaluation is conducted alongside a clinical trial.

Acknowledgements This research was performed within the framework of CTMM, the Center for Translational Molecular Medicine (http://www.ctmm.nl), projects TRIUMPH (Grant 01C-103) and ENGINE (Grant 01C-401), and supported by the Dutch Heart Foundation. The COACH study was supported by Grant $2000 \mathrm{Z} 003$ from the Dutch Heart Foundation and by additional unrestricted Grants from Biosite France SAS, Jouy-en-Josas, France (brain natriuretic peptide), Roche Diagnostics Nederland BV, Venlo, the Netherlands (N-terminal prohormone brain natriuretic peptide), and Novartis Pharma BV, Arnhem, the Netherlands.

Open Access This article is distributed under the terms of the Creative Commons Attribution 4.0 International License (http://creativeco mmons.org/licenses/by/4.0/), which permits unrestricted use, distribution, and reproduction in any medium, provided you give appropriate credit to the original author(s) and the source, provide a link to the Creative Commons license, and indicate if changes were made.

\section{References}

1. Briggs, A., Claxton, K., Sculpher, M.: Decision Modelling for Health Economic Evaluation. Oxford University Press, New York (2006)

2. Drummond, M.F., Sculpher, M.J., Claxton, K., Stoddart, G.L., Torrance, G.W.: Methods for the Economic Evaluation of Health Care Programmes, 4th edn. Oxford University Press, New York (2015)

3. Husereau, D., Drummond, M., Petrou, S., Carswell, C., Moher, D., Greenberg, D., Augustovski, F., Briggs, A.H., Mauskopf, J., Loder, E., ISPOR Health Economic Evaluation Publication Guidelines-CHEERS Good Reporting Practices Task Force: Consolidated Health Economic Evaluation Reporting Standards (CHEERS) — explanation and elaboration: a report of the ISPOR health economic evaluation publication guidelines good reporting practices task force. Value Health 16, 231-250 (2013)

4. Eichler, H.G., Kong, S.X., Gerth, W.C., Mavros, P., Jönsson, B.: Use of cost-effectiveness analysis in health-care resource allocation decision-making: how are cost-effectiveness thresholds expected to emerge? Value Health 7, 518-528 (2004)

5. Grutters, J., Sculpher, M., Briggs, A., Severens, J.L., Candel, M.J., Stahl, J.E., de Ruysscher, D., Boer, A., Ramaekers, B.L., Joore, M.A.: Acknowledging patient heterogeneity in economic evaluation. Pharmacoeconomics 31, 111-123 (2013)

6. Sculpher, M.: Subgroups and heterogeneity in cost-effectiveness analysis. Pharmacoeconomics 26, 799-806 (2008)
7. Willan, A., Briggs, A., Hoch, J.: Regression methods for covariate adjustment and subgroup analysis for non-censored cost-effectiveness data. Health Econ. 13, 461-475 (2004)

8. Coyle, D., Buxton, M.J., O’Brien, B.J.: Stratified cost-effectiveness analysis: a framework for establishing efficient limited use criteria. Health Econ. 12, 421-427 (2003)

9. Basu, A., Meltzer, D.: Value of information on preference heterogeneity and individualized care. Med. Decis. Mak. 27, 112-127 (2007)

10. Espinoza, M.A., Manca, A., Claxton, K., Sculpher, M.J.: The value of heterogeneity for cost-effectiveness subgroup analysis: conceptual framework and application. Med. Decis. Mak. 34, 951-964 (2014)

11. Claxton, K., Posnett, J.: An economic approach to clinical trial design and research priority-setting. Health Econ. 5, 513-524 (1996)

12. Tambour, M., Zethraeus, N., Johannesson, M.: A note on confidence intervals in cost-effectiveness analysis. Int. J. Technol. Assess. Health Care 14, 467-471 (1998)

13. Claxton, K.: The irrelevance of inference: a decision-making approach to the stochastic evaluation of health care technologies. J. Health Econ. 18, 341-364 (1999)

14. Hoch, J., Briggs, A., Willan, A.: Something old, something new, something borrowed, something blue: a framework for the marriage of health econometrics and cost-effectiveness analysis. Health Econ. 11, 415-430 (2002)

15. Bonetti, M., Gelber, R.: A graphical method to assess treatmentcovariate interactions using the cox model on subsets of the data. Stat. Med. 19, 2595-2609 (2000)

16. Bonetti, M., Gelber, R.: Patterns of treatment effects in subsets of patients in clinical trials. Biostatistics 5, 465-481 (2004)

17. Royston, P., Sauerbrei, W.: Interactions between treatment and continuous covariates: a step toward individualizing therapy. J. Clin. Oncol. 26, 1397-1399 (2008)

18. Jaarsma, T., van der Wal, M.H., Hogenhuis, J., Lesman, I., Luttik, M.L., Veeger, N.J., van Veldhuisen, D.J.: Design and methodology of the COACH study: a multicenter randomised coordinating study evaluating outcomes of advising and counselling in heart failure. Eur. J. Heart Fail. 6, 227-233 (2004)

19. Jaarsma, T., van der Wal, M.H., Lesman-Leegte, I., Luttik, M.L., Hogenhuis, J., Veeger, N.J., Sanderman, R., Hoes, A.W., van Gilst, W.H., Lok, D.J., Dunselman, P.H., Tijssen, J.G., Hillege, H.L., van Veldhuisen, D.J., Coordinating Study Evaluating Outcomes of Advising and Counseling in Heart Failure $(\mathrm{COACH})$ Investigators: Effect of moderate or intensive disease management program on outcome in patients with heart failure: coordinating study evaluating outcomes of advising and counseling in heart failure (COACH). Arch. Intern. Med. 168, 316-324 (2008)

20. Postmus, D., van Veldhuisen, D.J., Jaarsma, T., Luttik, M.L., Lassus, J., Mebazaa, A., Nieminen, M.S., Harjola, V.P., Lewsey, J., Buskens, E., Hillege, H.L.: The COACH risk engine: a multistate model for predicting survival and hospitalization in patients with heart failure. Eur. J. Heart Fail. 14, 168-175 (2012)

21. Rubin, D.: Multiple imputation after $18+$ years. J. Am. Stat. Assoc. 91, 473-489 (1996)

22. Postmus, D., Pari, A.A., Jaarsma, T., Luttik, M.L., van Veldhuisen, D.J., Hillege, H.L., Buskens, E.: A trial-based economic evaluation of 2 nurse-led disease management programs in heart failure. Am. Heart J. 162, 1096-1104 (2011)

23. Lazar, A.A., Cole, B.F., Bonetti, M., Gelber, R.D.: Evaluation of treatment-effect heterogeneity using biomarkers measured on a continuous scale: subpopulation treatment effect pattern plot. J. Clin. Oncol. 28, 4539-4544 (2010)

24. Boccardo, F., Guglielmini, P., Parodi, A., Rubagotti, A.: Chemotherapy versus tamoxifen versus chemotherapy plus tamoxifen in node-positive, oestrogen receptor-positive breast cancer 
patients. Very late results of the 'gruppo di ricerca per la chemioormonoterapia adiuvante (GROCTA)' 01-Trial in early breast cancer. Breast Cancer Res. Treat. 126, 653-661 (2011)

25. Demissei, B.G., Postmus, D., Liu, L.C.Y., Cleland, J.G., O’Connor, C.M., Metra, M., Ponikowski, P., Teerlink, J.R., Cotter, G., Davison, B.A., Edwards, C., Givertz, M.M., Bloomfield, D.M., Dittrich, H.C., Voors, A.A., Hillege, H.L.: Risk-based evaluation of efficacy of rolofylline in patients hospitalized with acute heart failure-post-hoc analysis of the PROTECT trial. Int. J. Cardiol. 223, 967-975 (2016)

26. Liu, L.C.Y., Valente, M.A.E., Postmus, D., O'Connor, C.M., Metra, M., Dittrich, H.C., Ponikowski, P., Teerlink, J.R., Cotter, G., Davison, B.A., Cleland, J.G., Givertz, M.M., Bloomfield, D.M., van Veldhuisen, D.J., Hillege, H.L., van der Meer, P., Voors, A.A.: Identifying subpopulations with distinct response to treatment using plasma biomarkers in acute heart failure: results from the PROTECT trial. Cardiovasc. Drugs Ther. 31, 281-293 (2017)

27. Pulignano, G., Del Sindaco, D., Di Lenarda, A., Tarantini, L., Cioffi, G., Gregori, D., Tinti, M.D., Monzo, L., Minardi, G.: Usefulness of frailty profile for targeting older heart failure patients in disease management programs: a cost-effectiveness, pilot study. J. Cardiovasc. Med. (Hagerstown) 11, 739-747 (2010)

28. DeBusk, R.F., Miller, N.H., Parker, K.M., Bandura, A., Kraemer, H.C., Cher, D.J., West, J.A., Fowler, M.B., Greenwald, G.: Care management for low-risk patients with heart failure: a randomized, controlled trial. Ann. Intern. Med. 141, 606-613 (2004)

29. Laramee, A.S., Levinsky, S.K., Sargent, J., Ross, R., Callas, P.: Case management in a heterogeneous congestive heart failure population: a randomized controlled trial. Arch. Intern. Med. 163, 809-817 (2003)

30. Chaudhry, S.I., Phillips, C.O., Stewart, S.S., Riegel, B., Mattera, J.A., Jerant, A.F., Krumholz, H.M.: Telemonitoring for patients with chronic heart failure: a systematic review. J. Card. Fail. 13, 56-62 (2007)

31. Hebert, P.L., Sisk, J.E., Wang, J.J., Tuzzio, L., Casabianca, J.M., Chassin, M.R., Horowitz, C., McLaughlin, M.A.: Cost-effectiveness of nurse-led disease management for heart failure in an ethnically diverse urban community. Ann. Intern. Med. 149, 540-548 (2008)

32. Miller, G., Randolph, S., Forkner, E., Smith, B., Galbreath, A.D.: Long-term cost-effectiveness of disease management in systolic heart failure. Med. Decis. Mak. 29, 325-333 (2009)

33. Goehler, A., Conrads-Frank, A., Worrell, S.S., Geisler, B.P., Halpern, E.F., Dietz, R., Anker, S.D., Gazelle, G.S., Siebert, U.: Decision-analytic evaluation of the clinical effectiveness and cost-effectiveness of management programmes in chronic heart failure. Eur. J. Heart Fail. 10, 1026-1032 (2008)

34. Burke, J.F., Hayward, R.A., Nelson, J.P., Kent, D.M.: Using internally developed risk models to assess heterogeneity in treatment effects in clinical trials. Circ. Cardiovasc. Qual. Outcomes 7, 163-169 (2014)

35. Kent, D.M., Rothwell, P.M., Ioannidis, J.P., Altman, D.G., Hayward, R.A.: Assessing and reporting heterogeneity in treatment effects in clinical trials: a proposal. Trials 11, 85 (2010)

36. Hayward, R.A., Kent, D.M., Vijan, S., Hofer, T.P.: Multivariable risk prediction can greatly enhance the statistical power of clinical trial subgroup analysis. BMC Med. Res. Methodol. 6, 18 (2006)

37. Rothwell, P.M.: Subgroup analysis in randomised controlled trials: importance, indications, and interpretation. Lancet 365, 176-186 (2005)

38. Royston, P., Altman, D.G., Sauerbrei, W.: Dichotomizing continuous predictors in multiple regression: a bad idea. Stat. Med. 25 , 127-141 (2006)

39. Cao, Q., Buskens, E., Feenstra, T., Jaarsma, T., Hillege, H., Postmus, D.: Continuous-time semi-Markov models in health economic decision making: an illustrative example in heart failure disease management. Med. Decis. Mak. 36, 59-71 (2016)

40. Phillips, K.A., Sakowski, J.A., Trosman, J., Douglas, M.P., Liang, S.Y., Neumann, P.: The economic value of personalized medicine tests: what we know and what we need to know. Genet. Med. 16, 251-257 (2014)

41. Jakka, S., Rossbach, M.: An economic perspective on personalized medicine. Hugo J. 7, 1 (2013)

42. Crawford, J.M., Aspinall, M.G.: The business value and costeffectiveness of genomic medicine. Pers. Med. 9, 265-286 (2012)

43. Postma, M.J., Boersma, C., Vandijck, D., Vegter, S., Le, H.H., Annemans, L.: Health technology assessments in personalized medicine: illustrations for cost-effectiveness analysis. Expert Rev. Pharmacoecon. Outcomes Res. 11, 367-369 (2011)

44. Conti, R., Veenstra, D.L., Armstrong, K., Lesko, L.J., Grosse, S.D.: Personalized medicine and genomics: challenges and opportunities in assessing effectiveness, cost-effectiveness, and future research priorities. Med. Decis. Mak. 30, 328-340 (2010)

45. Cao, Q.: Towards Optimal Decision Making in Personalized Medicine: Potential Value Assessment of Biomarkers in Heart Failure Exemplars. Ridderprint BV, Ridderkerk (2015)

46. Yu, T., Vollenweider, D., Varadhan, R., Li, T., Boyd, C., Puhan, M.A.: Support of personalized medicine through risk-stratified treatment recommendations - an environmental scan of clinical practice guidelines. BMC Med. 11, 7 (2013) 